\title{
A Ética e os Custos Sociais da "Reforma Psiquiátrica"
}

\author{
THE ETHICS AND THE SOCIAL COSTS OF THE \\ "PSYCHIATRIC REFORM"
}

Valentim Gentil ${ }^{(*)}$

"Com a Lei n. 180, pela primeira vez no mundo ocidental, todos os cidadãos readquirem pleno direito de cidadania política e civil."(1)

"... it is not difficult to diminish the number of hospital inmates if one does not care for the discharged chronic patients and fails to secure their welfare."(2)

"Esse processo de transição requer planejamento competente ... exporá, com certeza, muitas feridas sociais. Esta exposição será moralmente atribuída aos gestores da reforma e isso implicará numa discussão ética que devemos prever."(3)

\section{RESUMO}

Este artigo aborda questões relevantes sobre a Lei n. 10.216/01, que provocou a chamada "Reforma Psiquiátrica" no Brasil, bem como as leis estaduais em conseqüência da lei federal, ainda mais radicais no que se refere à desospitalização, determinando até mesmo a proibição de leitos hospitalares destinados a doentes mentais. O Autor aponta a drástica redução do número de leitos, em contraste com o aumento populacional num espaço de 10 anos, sem oferecer contrapartidas de atendimento adequado, deixando aqueles que necessitam de tratamento, abandonados à própria sorte, presos em suas casas ou "morando" nas ruas. Critica essa desos-

(*) Professor Titular de Psiquiatria da Faculdade de Medicina da USP, Presidente do Conselho Diretor do Instituto de Psiquiatria do HC-FMUSP e Ph. D. pelo Instituto de Psiquiatria da Universidade de Londres. E-mail: vgentil@usp.br. Entrada: 29.11.03 _Aprovado em 20.1.2004.

(1) ROTELLI, Franco. A Lei n. 180 da reforma psiquiátrica: os problemas na sua aplicação. In: ENCONTRO ITALO-BRASILEIRO DE SAÚDE, 18-21 jun. 1989, Salvador (BA), Saúde mental: a reforma psiquiátrica em questão. Anais ..., Salvador, 1989.

(2) BLEULER, Manfred. In: SHEPHERD, M. (Ed.). "Psychiatrists on psychiatry". London: Cambridge University Press, 1982. pp. 1-13.

(3) FIGUEIREDO, Gabriel. "Ética e reforma da assistência psiquiátrica no Brasil". Temas, n. 60/ 61, pp. 1-14, 2001. 
pitalização imposta pela norma sem a implementação de recursos alternativos eficientes para atender àqueles que necessitam de atendimento e tratamento, adotando-se uma fórmula comprovadamente fracassada nos países que tentaram implantar o modelo sem prover, simultaneamente, recursos alternativos comensuráveis. Aponta para a importância da participação interdisciplinar da Universidade, contribuindo para a busca de soluções para este grave problema médico-social e para a necessidade de investimentos nas prevenções primária e secundária como forma de reduzir a incapacitação do doente mental, bem como no treinamento técnico atualizado das equipes que trabalham com saúde mental.

\section{Descritores}

Reforma psiquiátrica; Política de Saúde; Saúde Mental; Hospitais Psiquiátricos; Desospitalização; Leitos Hospitalares; Recursos Alternativos; Custo Social.

\section{ABSTRACT}

This article addresses questions related to Brazilian Law 10.216/01 and the State laws of the so-called "Brazilian Psychiatric Reform". Desospitalization resulted from the closure of more than 60 thousand psychiatric beds in 20 years. The author criticizes this policy, implemented without the provision of commensurate alternative resources, also proven unsuccessful in other countries. An urgent priority of investments in primary and secondary prevention is advocated to reduce the burden of mental illness, with proper training of mental health workers and education of society at large. The importance of an interdisciplinary effort by the University, in the search for solutions for this grave medical and social problem, is highlighted.

\section{Keywords}

Psychiatric Reform; Politic as of Mental health; Psychiatric Hospitals; Desospitalization; Alternative Resources; Social Cost.

Os transtornos psiquiátricos são variados e não se restringem às psicoses e aos quadros mais crônicos, objeto principal das políticas públicas no Brasil desde os seus primórdios. Um bom Sistema de Saúde Mental deve ser, hoje, baseado em informações epidemiológicas confiáveis, abrangente, hierarquizado e com clara identificação de prioridades. Estudos epidemiológicos bem conduzidos indicam que, excluindo-se a dependência de nicotina, mais de $30 \%$ da nossa população sofrerá em suas vidas de pelo menos um transtorno mental dentre os constantes da Classificação Internacional de Doenças, $(\mathrm{CID}-10)^{(4)}$. Essas taxas são semelhantes às encon-

(4) ANDRADE, L.; WALTERS, E. E.; GENTIL, V.; LAURENTI, R. "Prevalence of ICD-10 mental disordes in a catchment area in the city of São Paulo", Brazil. Social Psychiatry and Psychiatric Epidemiology, n. 37, pp. 316-325, 2002. 
tradas em países desenvolvidos e é impossível atender a toda a demanda decorrente desses transtornos, principalmente nos países menos desenvolvidos. ${ }^{(5)}$

A se confirmarem as indicações de crescente prevalência das doenças mentais graves, o que foi recentemente considerado equivalente a “Uma Peste Invisível" (6), a Sociedade terá que investir de forma mais inteligente na prevenção primária possível, oferecer prevenção secundária eficaz e com melhor relação custo/benefício, reduzindo a incapacitação, e distribuir seus limitados recursos de acordo com uma lógica que pondere o sofrimento, a sobrecarga e a eficiência dos procedimentos disponiveis ${ }^{(7)}$. Isto não aconteceu na assim chamada "reforma psiquiátrica brasileira" (8), mais engajada em movimentos sociais do que voltada para o efetivo atendimento às necessidades dos que sofrem doença mental. Abordei aspectos desta questão ${ }^{(9)}$ quando da votação da Lei n. 10.216, de 6.4.2001, que "Dispõe sobre a proteção e os direitos das pessoas portadoras de transtornos mentais e redireciona o modelo assistencial em saúde mental". Em se tratando de um tema de grande relevância e complexidade, isto deveria ser objeto de um denso projeto interdisciplinar da Universidade, que não mobilizou ainda, de forma coordenada, a sua capacidade de contribuir para a busca de soluções para este grave problema médico-social.

Depois de mais de uma década tentando entender a lógica e os motivos que levaram à nossa "desospitalização", exemplo local do que foi considerado "o experimento social mais fracassado do Século XX"(10), acho importante rever por quê, como e a que custo social se aplica aqui um modelo "basagliano"(11) de "reforma psiquiátrica". Nesse sentido, lembro que o pro-

(5) WORLD MENTAL HEALTH SURVEY CONSORTIUM. "Prevalence, severity, and unmet need for treatment of mental disorders in the World Health Organization World Mental Health Surveys". JAMA, n. 291, pp. 2581-2590, 2004.

(6) TORREY, E. Fuller; MILLER, Judy. "The invisible plague: the rise of mental illness from 1750 to the present". London: Rutgers University Press, 2001, 416 p. Eles demonstram um aumento de 9 vezes na prevalência de doenças mentais graves nos Estados Unidos entre 1880 e 1980 , mas não encontram explicação satisfatória para isto.

(7) ANDREWS, G.; HENDERSON, S. "Unmet needs in psychiatry: problems, resources, responses". London: Cambridge University Press, 2000.

(8) Contrariamente aos modelos assistenciais, as especialidades médicas podem ser criadas, desenvolvidas, extintas, mas não "reformadas".

(9) GENTIL, V. "Uma leitura anotada do projeto brasileiro de 'Reforma Psiquiátrica'". Revista USP, São Paulo, n. 43, pp. 6-23, 1999.

(10) TORREY, E. Fuller. "Jails and prisons: America's new mental hospitals". American Journal of Public Health, n. 5, pp. 1611-1613, 1995.

(11) Franco Basaglia e seguidores vieram ao Brasil algumas vezes desde 1978, a convite de líderes do MTSM (depois ampliado para Movimento da Luta Antimanicomial). De acordo com Benedetto Saraceno, hoje Diretor da Divisão de Saúde Mental e Abuso de Substâncias da Organização Mundial da Saúde, em Genebra, foi a partir da, mal conhecida mas muito citada, "Conferência de Caracas" que o movimento basagliano criou "um consórcio informal para apoiar paises como o Brasil". Segundo Saraceno, suas "intervenções" ocorrem em 5 níveis ("serviços psiquiátricos; administrativo; docente; legislativo; político"). Os consorciados eram Franco Rotelli, 
blema é milenar e a proposta de defesa de direitos não é nova. Assim se expressou Teixeira Brandão há 90 anos: "Os alienados eram enviados para o asilo de mendigos ... ou para as cadeias ... Em regra geral, esses infelizes ficavam ao abandono, ou eram reclusos nas cadeias públicas. ... O que compete à autoridade é que a lei impeça as seqüestrações arbitrárias, que não permita a reclusão senão depois das provas documentadas da moléstia, impossibilitando dessa arte as fraudes e as violências contra a liberdade individual sob pretexto de uma moléstia que, na realidade, não existe"(12).

Em quê o atendimento às necessidades dos portadores de transtornos mentais melhorou no Brasil desde Teixeira Brandão? A crítica de Manfred Bleuler se aplica ao que aqui se faz desde $1985^{(13)}$ ? Qual a implicação ética de insistir em uma política cuja evidente inviabilidade era conhecida desde antes da sua implantação? Essas são questões obrigatórias para os responsáveis pelas políticas de Saúde e para os que zelam pelos Direitos Humanos.

Em relação à época de Teixeira Brandão, a salvaguarda dos direitos e a operacionalização dos procedimentos para controle das internações involuntárias, foram mais explicitados pela Lei n. 10.216, de 6.4.2001. É importante notar que, apesar da sua ementa mencionar um redirecionamento do modelo assistencial, essa Lei não contém a exigência basagliana de fechar leitos e proibir novos hospitais psiquiátricos, ao contrário do "Projeto Delgado" ${ }^{(14)}$ e da maioria das Leis Estaduais apresentadas pelo

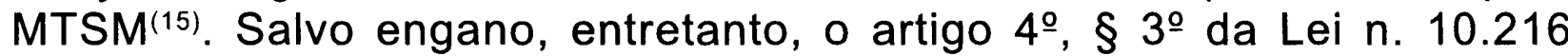

\footnotetext{
Ernesto Venturini e Manuel Desviat (SARACENO, B. II progetto dell'Istituto Mario Negri: Salute Mentale in America Latina. "Epidemiologia e Psichiatria Sociale", n. 3, pp. 45-68, 1994). "Caracas" foi uma reunião sem maior representatividade, mas sua "Declaração" é utilizada como se fosse um documento oficial. Escrevi isto em um Editorial (GENTIL, V. "Projeto Delgado", Substitutivo Rocha ou uma Lei melhor? Revista Brasileira de Psiquiatria, n. 23, pp. 1-2, 2001). Fui contestado por Levav, ex-funcionário da OPAS e um dos organizadores da reunião de Caracas. Pude então dar mais detalhes em apoio às minhas afirmações (LEVAV, Itzhak; GENTIL, Valentim. "Posturas ideológicas contrastan con las respuestas cientificas a las necesidades de salud mental de las poblaciones del Brasil y delas Américas". Rev. Bras. Psiquiatria, n. 23, pp. 117-118, 2001).
}

(12) BRANDÃO, Teixeira. "Elementos fundamentaes de psychiatria clinica e forense". Rio de Janeiro: Leite Ribeiro \& Maurillo, 1918, p. 139. Brandão é considerado o primeiro grande nome da Psiquiatria brasileira (Leme Lopes, 1988, entrevista ao projeto "Memória Viva da Psiquiatria Brasileira"; videoteca do Instituto de Psiquiatria do HC-FMUSP). Na mesma época, Francisco Franco da Rocha exercia influência semelhante em São Paulo.

(13) Ano em que o Movimento dos Trabalhadores em Saúde Mental (MTSM) começou a exercer o controle da área de saúde mental pública no Brasil: "A partir de 1985, ... uma parte significativa dos postos de chefia de programas estaduais e municipais de saúde mental, assim como a direção de importantes unidades hospitalares públicas - inclusive algumas universitárias estão sob a condução de fundadores e ativistas do MTSM" (AMARANTE, P. e cols. "Loucos pela vida: a trajetória da reforma psiquiátrica no Brasil". Rio de Janeiro: Fiocruz, 1995, 136 p.).

(14) Cujo artigo 19 rezava: "Fica proibida, em todo o território nacional, a construção de novos hospitais psiquiátricos públicos e a contratação ou financiamento, pelo setor governamental, de novos leitos em hospital psiquiátrico".

(15) As leis estaduais em vigor são: Ceará, Lei n. 12.151 (29.7.1993), sancionada pelo Governador Ciro Ferreira Gomes (PL de 1992, de Mario Mamede - PT); Espírito Santo, Lei n. 5.267 
comete o equívoco de proibir o acesso de portadores de transtorno mental ao Asilo, em vez de proibir o Hospital Asilar, mantendo a confusão entre equipamentos médicos e de cunho social na área psiquiátrica e discriminando aqueles que, não mais precisando de cuidados hospitalares, poderiam contar com as tradicionais funções de abrigo e proteção oferecidas pelos asilos.

Quanto ao "abandono", a evidência está escandalosamente exposta nas ruas das nossas cidades, mas não está ainda dimensionada e pode ser muito maior. Em maio de 2003, o Coordenador de Saúde Mental do Ministério da Saúde (Pedro Delgado) declarou que os serviços disponiveis “... acolhem no máximo 1 milhão de doentes. Os outros 4 milhões estão trancados em suas casas. São vistos perambulando pelas ruas ou, quando têm um surto mais grave, são levados para as emergências dos hospitais - ou para as delegacias ... Faltam dinheiro e equilíbrio nas ações"(16).

$\mathrm{Na}$ ausência de estudos especializados, é impossível dimensionar a "desassistência" no país. Um Doutorado defendido no Departamento de Psiquiatria da FMUSP, revela os seguintes diagnósticos, feitos pessoalmente por um psiquiatra experiente, entre 83 adultos moradores de rua há pelo menos 1 ano, em Juiz de Fora (MG): alcoolismo (82\%), abuso de drogas $(31 \%)$, transtornos do humor $(32,5 \%)$, psicoses esquizofreniformes $(9,6 \%)$, combinações de transtornos mentais $(78 \%)$. Apenas uma pessoa não tinha transtorno mental pela CID-10(17).

Segundo o site da Secretaria Municipal de Assistência Social (acesso em 8.11.2004), o recenseamento realizado pela FIPE em outubro de 2003 encontrou 10.394 (mais de $80 \%$ homens) "pessoas em situação de rua na área pesquisada" no município de São Paulo (um crescimento de $100 \%$ em relação à estimativa de 4.549 moradores em 1994). Dessas, 4.208 eram "Moradores de Rua" e 6.186 "Moradores Albergados"(18). Reportagem do Estado de São Paulo em 22.8.2004, à época dos recentes e

(10.9.1996), sancionada pelo Governador Vitor Buaiz (PL de 1995, de Lelo Coimbra.), Minas Gerais, Lei n. 11.802 (18.1.1995), sancionada pelo Governador Eduardo Azeredo (PL de 1992, de Antonio Carlos Pereira - PT); Paraná, Lei n. 11.189 (9.11.1995) sancionada pelo Governador Jaime Lerner (PL de 1995, de Florisvaldo Fier "Rosinha" - PT); Pernambuco, Lei n. 11.064 (16.5.1994), sancionada pelo Governador Joaquim Francisco (PL de 1993, de Humberto Costa - PT); Rio Grande do Norte, Lei n. 6.758 (4.1.1995), promulgada pelo Presidente da Assembléia Legislativa Dep. Raimundo Fernandes (PL de Lauro Bezerra - PMDB e Júnior Souto PT); Rio Grande do Sul, Lei n. 9.716 (7.8.1992), sancionada pelo Governador Alceu Collares (PL de 1991, de Marcos Rolim - PT); e Distrito Federal, (PL de 1997, de Adão Xavier - PPB).

(16) Número de internações cai no país, mas especialistas reclamam da falta da verba. Folha de S. Paulo, São Paulo, 18 maio 2003. Cotidiano.

(17) HECKERT, U. e cols. "Lifetime prevalence of mental disorders among homeless people in a southeast city in Brazil". European Archives of Psychiatry and Clinical Neurosciences, n. 249, pp. 150-155, 1999.

(18) Um dos primeiros atos da gestão Suplicy foi a assinatura do Decreto n. 40.232, de 2.1.2001 regulamentando a Lei n. 12.316, de 16.4.1997 sobre a obrigatoriedade do Poder Público Municipal prestar atendimento à população de rua. Infelizmente, nem a Lei nem o Decreto e nem o Censo mencionam os graves transtornos mentais dessa população. 
brutais assassinatos, afirma que: "Na cidade, não há albergues para todos. E mesmo nesses locais tudo se resume a tirar mendigos do espaço público, dar-lhes banho, refeição, e oferecer-Ihes uma cama para, às 6 ou 7 horas, jogá-los na rua de novo".

É importante reconhecer os esforços realizados para o atendimento e a melhoria das condições de habitação, segurança, apoio humanitário e até lazer da população de rua, especialmente nos últimos quatro anos. Entretanto, é errôneo abordar os problemas do "Povo da Rua" ou dos "Sem-Teto", incluindo os que estão em favelas e cortiços, sem atender medicamente as pessoas com graves e incapacitantes doenças mentais. Se as prevalências de Juiz de Fora forem aplicáveis a São Paulo (aqui tudo é mais grave), mais de 1.000 pessoas nas ruas há mais de 1 ano estão psicóticas e sem tratamento. Não fossem os governos os responsáveis pela falta de recursos para atendimento psiquiátrico a essa população, estaríamos nós, os profissionais de saúde, diariamente infringindo a Ética, por omissão de socorro ao passar por esses doentes ${ }^{(19)}$.

Pouco consola saber que outros paises têm problema semelhante e que nem todos os psicóticos do "povo da rua" são ex-pacientes despejados pelo fechamento dos "manicômios"(20). Estudos em outros países demonstraram que são os novos psicóticos que, não atendidos pelo sistema de saúde, acabam nas ruas. Faltam leitos e recursos alternativos. O mais grave é que aqui se sabia que isto iria acontecer. De fato, em 1989, Franco Rotelli, sucessor de Basaglia em Trieste (cidade peculiar por sua localização e história, então com menos de 300.000 habitantes), disse em "Encontro" na Bahia(21), que a "política de aumentar os serviços externos para reduzir os hospitais psiquiátricos e oferecer melhores possibilidades de cura foi considerada um fracasso por todos os governos da Europa Ocidental ... Passados 30 anos, podemos dizer tranqüilamente que esta política faliu. Faliu na Inglaterra, na França, nos Países Escandinavos e depois também na Alemanha" (p. 119). Depois de sugerir que o "fechamento de $70 \%$ dos hospitais psiquiátricos nos Estados Unidos foi realizado sobretudo para reduzir as despesas do Estado" e chamar essa política "pelo simples slogan: 'os loucos pelas ruas', Rotelli disse que a literatura científica internacional levava a uma "terrível mistificação" ao não discriminar entre a situação italiana em geral, de "os loucos para as ruas" e aquela de regiões onde a reforma italiana foi efetivada pelo "movimento liderado por Franco Basaglia" (p. 120).

(19) Em 1987 um articulista do New York Times reclamou: "If your leg is broken, the city will take you away: if your mind is broken, you just lie there forever" (citado por ISAAC, R. J.; ARMAT, V. C. Madness in the streets: how psychiatry and the law abandoned the mentally ill. New York: The Free Press, 1990, p. 2).

(20) A diferença entre Manicômio, Asilo e Hospital é sobejamente conhecida, mas a utilização do termo Manicômio para se referir a todos os hospitais psiquiátricos foi uma decisão "estratégica" do MTSM (AMARANTE, P. e cols., op. cit., p. 95).

(21) Vide nota 1. 
Compilando, todavia, essa "literatura científica", verifica-se que os que analisaram a reforma italiana sempre disseram que ela foi bem sucedida apenas onde haviam projetos-piloto e onde a proposta foi fortemente subsidiada(22). Tudo isto foi documentado ad nauseam, antes, durante e depois da tramitação do projeto Delgado no Congresso Nacional. Mas aqui, também, não se seguiu este aspecto da recomendação de Basaglia ou Rotelli.

Poder-se-ia supor que alguns foram iludidos por Rotelli, em Salvador, que disse que estariam sendo "destinados 1 bilhão de dólares para implementar, nos próximos três anos, uma parte dos serviços alternativos ao hospital psiquiátrico ... a construção de 250 comunidades para os exinternos... e 200 novos serviços residenciais de reabilitação... além de 600 comunidades de hospedagem para de 5 a 7 pessoas perfazendo 4.000 vagas" (p. 120). Pura ilusão. Onze anos depois, dois psiquiatras do Serviço Nacional de Saúde da Itália, De Girolamo e Cozza ${ }^{(23)}$, contaram que pouco se sabe sobre o destino dos pacientes desospitalizados na Itália(24) e que as famílias arcavam com grande sobrecarga pois assumiram responsabilidades que antes eram do governo. Talvez por isto exista um movimento para reverter as conseqüências da Lei $n .180$ naquele país ${ }^{(25)}$.

Quanto ao comentário de Bleuler, basta cotizar os textos e cifras oficiais. Assim, na "Justificação" do Projeto de Lei n. 3.675, submetido à Câmara dos Deputados em 12.9.1989, o Deputado Paulo Delgado afirmava: "No Brasil, os efeitos danosos da política de privatização paroxística da saúde, nos anos 60 e 70, incidiram violentamente sobre a saúde mental, criando um parque manicomial de quase 100.000 leitos remunerados pelo setor público, além de cerca de 20.000 leitos estatais"(26). Em 19.8.2004, o site do Ministério da Saúde trazia as seguintes informações: "O Brasil tem atualmente 47.843 leitos psiquiátricos. Em 2000, eram 60.868. Entre janeiro do ano passado e julho de 2004, o Ministério da Saúde já reduziu 4.627 leitos. Nos anos 1970, o país chegou a ter mais de 100 mil leitos psiquiátricos. Até o primeiro semestre de 2005, a meta é extinguir mais 3,5 mil leitos".

A incongruência entre o discurso e a ação fica evidente ao confrontar a realidade com o que diz o texto do "Programa Anual de Reestruturação da Assistência Hospitalar no SUS - 2004" (Portaria do Ministério da Saúde, 52/GM, de 20.1.2004): "O processo de mudança do modelo assistencial deve ser conduzido de modo a garantir uma transição segura, onde a

(22) Por exemplo, "... nothing uniquely Italian about this experience. In Britain, the USA, and Australia ... the same mix of successful local services (often set up as pilot schemes and part of a research programme) and the same failure on a large enough scale" (CRAIG, T. The 1978 Italian mental health law - a personal evaluation. Br. J. Psychiatry, n. 159, p. 560, 1991).

(23) DE GIROLAMO, G.; COZZA, M. "The Italian psychiatric reform - a 20-year perspective. International Journal of Law and Psychiatry, n. 23, pp. 197-214, 2000.

(24) Aqui também não sabemos para onde foram os pacientes ou os recursos financeiros "assim economizados".

(25) Vide, por exemplo, matéria intitulada "La 180 è da riformare - tornano i mini-manicomi", no jornal La Republica, 21 set. 2001.

(26) Diário do Congresso Nacional (Seção I), em 27 de junho de 1990. 
redução dos leitos hospitalares possa ser planificada e acompanhada da construção concomitante de alternativas de atenção no modelo comunitário". Foram extintos mais de 60 mil leitos num período em que a população passou de 80 para 180 milhões de habitantes e nada comensurável foi feito de recursos alternativos. Mantida a mesma relação leito/habitantes, o Brasil teria hoje mais de 200 mil leitos e tem menos de 1/4 disso. Portanto, a redução na disponibilidade de leitos psiquiátricos para a população foi efetivamente de $75 \%{ }^{(27)}$. Aliás, essa mesma ambigüidade de discurso está presente na Lei n. 11.064 (16.5.1994), decorrente do Projeto de Lei assinado pelo Ministro Humberto Costa quando era Deputado Estadual em Pernambuco ${ }^{(28)}$. Na prática os leitos foram fechados e os recursos assistenciais alternativos foram irrisórios e tardios, como se pode verificar no site do Ministério da Saúde.

O site continua: "O Ministério da Saúde calcula que existem pelo menos 15 mil internos em instituições hospitalares psiquiátricas que poderiam retornar imediatamente ao convívio social ... Atualmente o programa (De Volta Para Casa) atende a 520 pessoas, que recebem uma bolsa de $R \$ 240$ por mês; Residências Terapêuticas - são $220 \mathrm{em}$ todo o país, nas quais moram mais de 2 mil pessoas; Centros de Atenção Psicossocial (Caps) - são $546 \mathrm{em}$ todo o país, dos quais 64 especificamente para o tratamento de dependentes de álcool e drogas e 41 voltados para crianças e adolescentes. O número de atendimentos nos Caps, que em 2002 foi de 389 mil, no ano passado chegou a 3,7 milhões - quase dez vezes maior". Ou seja, a desproporção entre o fechamento de leitos e a criação de recursos alternativos é enorme. Esse espetacular crescimento no atendimento dos Caps, sem aumento correspondente em suas unidades, aguarda explicação convincente.

Não sabemos quantos doentes mentais graves estão em nossas cadeias e prisões. Supõe-se que sejam milhares ${ }^{(29)}$. Colegas especializados em Psiquiatria Forense me dizem que esses estudos não existem. Sabe-se que nos países que passaram por experiências semelhantes como a Itália, a Inglaterra e os Estados Unidos, o panorama é desolador. Centenas de milhares de doentes mentais estão detidos ou condenados pela justiça, a maioria sem atenção psiquiátrica e submetidos a sofrimento extraordinário em conseqüência de sua incapacidade de entender e responder adequa-

(27) A mesma taxa criticada por Rotelli em sua análise da "reforma" americana no Encontro de 1989 na Bahia.

(28) O artigo 2ำ reza: "O Estado de Pernambuco substituirá progressivamente, mediante planificação anual, os leitos dos hospitais psiquiátricos pelos recursos assistenciais alternativos definidos nesta Lei". Já, o artigo 9 diz: "Fica proibida ao Estado de Pernambuco, por sua administração direta, fundações, autarquias, empresas públicas, sociedades de economia mista, nas quais detenha participação acionária, construir, ampliar, contratar ou financiar novos estabelecimentos, instituições privadas ou filantrópicas que caracterizem hospitais psiquiátricos" .

(29) O censo penitenciário de 1997, concluido em 1998, encontrou 170.602 presos no país, e 67.786 no Estado de São Paulo. TABORDA, J. G. V.; CARDOSO, R.G.; MORANA, Cardoso, H. C. P. Forensic psychiatry in Brazil an overview. International Journal of Law and Psychiatry, n. 23, pp. 579-588, 2000. 
damente às exigências disciplinares das instituições correcionais ${ }^{(30)}$. Em seu forte artigo de 1995 sobre os doentes mentais graves em cadeias e prisões americanas, Fuller-Torrey diz que “... 30\% dos detidos em cadeias aguardam avaliação ou transferência para hospitais"(31).

Em países onde não há atenuação da responsabilidade penal para atos praticados sob grave transtorno mental, casos como o da enfermeira Andrea Yates, destaque da revista Times de 13.7.2001, hoje em prisão perpétua pelo assassinato dos seus 5 filhos pequenos durante estado depressivo pós-parto, subtratado, suscitam reflexões sobre quem deveria ser condenado: o doente ou o Sistema que impede seu tratamento adequado ${ }^{(32)}$ ?

Em relação às crianças, uma das áreas de maior investimento e progresso da moderna Psiquiatria, a Secretaria de Estado da Saúde lançou, em 2003, "Uma proposta de Organização da Atenção - Política de Saúde Mental para a Infância e a Adolescência" e vários serviços universitários, notadamente em São Paulo e em Porto Alegre, estão desenvolvendo importante atividade de pesquisa em colaboração internacional de alto nível e oferecem assistência bem qualificada à população. Na maioria dos centros, entretanto, crianças psicóticas, com Transtorno Bipolar do Humor, depressivas, com fobias ou Transtorno Obsessivo-Compulsivo, sofrem durante anos até receberem diagnóstico e tratamento adequado. Enquanto isso, professores e profissionais de saúde treinados inadequadamente tentam "entender" seus comportamentos francamente alterados, como se fossem passiveis de modificação sem a moderna Psiquiatria Infantil|(33).

Em síntese, uma releitura da "trajetória da reforma" leva à suposição de que os responsáveis pelo atual modelo, desiludidos com as péssimas condições dos manicômios deteriorados, engajados em movimentos sociais e compondo-se com profissionais sequiosos por melhores condições de trabalho ou que desejavam implementar propostas de reforma sanitária priorizando cuidados primários, acabaram comprometendo-se com uma ideologia anacrônica e um modelo inviável economicamente.

(30) Recente informativo eletrônico atualiza esse número: "According to one estimate, on any given day, at least 284,000 people with schizophrenia and bipolar are incarcerated in the US, and 547,800 are on probation." "Prisons as mental hospitals". McMan's Depression and Bipolar Weekly, v. 5, n. 28, Oct. 31, 2003.

(31) TORREY, E. F., op. cit., pp. 1611-1613.

(32) Psicoses puerperais respondem melhor à eletroconvulsoterapia (ECT) do que a qualquer outro tratamento conhecido, mas a prescrição de ECT no Texas, encontra forte resistência e desinformação. Entre nós, apesar desse tratamento não estar disponível de forma tecnicamente adequada na maioria absoluta da rede pública e nem ser remunerado pelo SUS, existem serviços bem qualificados, em geral nas universidades.

(33) O relato dramático a seguir foi extraído do New York Times Magazine de 31.10.2004: "The suffering of mentally ill children who do not get treatment can be overwhelming ... They've got voices telling them what to do," said Ms. Nayowith. "They've got hallucinations. They have anxiety disorders, which means they can never sit and relax, or obsessive-compulsive disorders hand-washing, pacing. They have phobias and horrible eating disorders. Some are cutters you know, they mutilate themselves". 
Sem recursos para implementá-lo adequadamente, optaram pela desospitalização que a literatura e seus próprios mentores alertavam ter sido catastrófica em todos os Continentes.

Apesar dos elevados custos sociais previstos, eles tentaram aprovar projetos de Lei com textos tão radicais como o que reza: "... Ficam desautorizados a funcionar no território estadual todos os hospitais psiquiátricos ou similares existentes após 5 anos da data de promulgação desta Lei" (PL n. 366, AL de São Paulo, 19.5.92, Dep. R. Gouveia e outros). Antes mesmo que conseguissem aprovar tais leis, representantes do MTSM em vários Estados e na Coordenadoria de Saúde Mental do Ministério da Saúde diziam que não, mas fechavam milhares de leitos, sem que recursos alternativos estivessem disponíveis ${ }^{(34)}$. Alegava-se que "com o dinheiro assim economizado" seria suficiente para criar uma rede de recursos alternativos e reabilitação para pessoas com seqüelas incuráveis. Deixaram de investir na prevenção secundária para impedir as seqüelas, fechando ambulatórios psiquiátricos estaduais antes que os municípios estivessem habilitados para substituí-los e impediu-se a modernização ou criação de modernos hospitais especializados, talvez por que isto significaria reconhecer a eficácia da moderna Psiquiatria em vez de promover sua "desconstrução".

Mais recentemente, o Ministério da Saúde passou a utilizar um instrumento derivado de uma obscura "... pesquisa da satisfação do cliente externo , já realizada no Grupo Hospitalar Conceição, e desenvolvido pela FUNDATEC" para a classificação e remuneração diferencial dos serviços hospitalares, no Pnash $^{(35)}$. Através disso e da menor remuneração quanto maior o número de leitos oferecidos, independentemente da qualidade e resolutividade dos serviços, a política de fechamento de hospitais antes que eles se tornassem desnecessários, rejeitada no Congresso Nacional, continua a ser implementada.

Como se não estivéssemos em crise de efetividade, continua-se a referendar discutíveis progressos do atual "modelo", e utiliza-se textos ultrapassados e de baixa qualidade técnica ${ }^{(36)}$ para a "formação" de equipes de saúde mental.

(34) “... loucura? É um transtorno transitório ou não das relações da pessoa com seu meio. É uma doença relativa ... o projeto de Lei do deputado Paulo Delgado (PT) ... propõe ... a mudança do modelo assistencial ... já está sendo implementado ... Ela não propõe o desmantelamento da assistência psiquiátrica mas a substituição gradual por outras formas de cuidado." LOUCURAS que se realizam. Jornal do Brasil, Rio de Janeiro, 8 jan. 1995. Entrevista com Domingos Sávio, então Coordenador de Saúde Mental do Ministério da Saúde.

(35) Programa Nacional de Avaliação dos Serviços Hospitalares (Portaria n. 251, GM 31.1.2002). Esse instrumento considera excelentes os serviços que "Não realizam ECT" e nos quais o "Tempo médio de internação não ultrapassa 15 dias", entre outras negações da realidade dos pacientes com quadros mais graves.

(36) Por exemplo: SARACENO, B. Libertando identidades: da reabilitação psicossocial à cidadania possivel. Rio de Janeiro: Instituto Franco Basaglia - Te Corá Ed., 1999. 
Em conclusão, não tenho dúvida que a Ética e a Sociedade exigem uma imediata "Reforma mais Psiquiátrica da Política de Saúde Mental no Brasil", que garanta direitos, inclusive a paridade na cobertura financeira dos planos de Saúde, garanta acesso a recursos terapêuticos eficazes de forma efetiva, e exija uma atuação eficiente em todos os niveis de prevenção. Isto não será possível sem o abandono da ideologia antipsiquiátrica que pretende o contrário.

\section{REFERÊNCIAS BIBLIOGRÁFICAS}

AMARANTE, P. e cols. "Loucos pela vida: a trajetória da reforma psiquiátrica no Brasil". Rio de Janeiro: Fiocruz, 1995, 136 p.

ANDRADE, L.; WALTERS, E. E.; GENTIL, V.; LAURENTI, R. "Prevalence of ICD-10 mental disordes in a catchment area in the city of São Paulo", Brazil. Social Psychiatry and Psychiatric Epidemiology, n. 37, pp. 316325, 2002.

ANDREWS, G.; HENDERSON, S. "Unmet needs in psychiatry: problems, resources, responses". London: Cambridge University Press, 2000.

BLEULER, Manfred. In: SHEPHERD, M. (Ed.). "Psychiatrists on psychiatry". London: Cambridge University Press, 1982.

BRANDÃO, Teixeira. "Elementos fundamentaes de psychiatria clinica e forense". Rio de Janeiro: Leite Ribeiro \& Maurillo, 1918.

CRAIG, T. "The 1978 Italian mental health law - a personal evaluation". Br. J. Psychiatry, n. 159, 1991.

DE GIROLAMO, G.; COZZA, M. "The Italian psychiatric reform - a 20year perspective". International Journal of Law and Psychiatry, n. 23, pp. 197-214, 2000.

FIGUEIREDO, Gabriel. "Ética e reforma da assistência psiquiátrica no Brasil". Temas, n. 60/61, pp. 1-14, 2001.

GENTIL, V. "Uma leitura anotada do projeto brasileiro de 'Reforma Psiquiátrica". Revista USP, São Paulo, n. 43, pp. 6-23, 1999.

"Projeto Delgado, Substitutivo Rocha ou uma Lei melhor?" Revista Brasileira de Psiquiatria, n. 23, pp. 1-2, 2001.

HECKERT, U. e cols. "Lifetime prevalence of mental disorders among homeless people in a southeast city in Brazil". European Archives of Psychiatry and Clinical Neurosciences, n. 249, pp. 150-155, 1999.

ISAAC, R. J.; ARMAT, V. C. "Madness in the streets: how psychiatry and the law abandoned the mentally ill". New York: The Free Press, 1990. 
LEVAV, Itzhak; GENTIL, Valentim. "Posturas ideológicas contrastan con las respuestas científicas a las necesidades de salud mental de las poblaciones del Brasil y delas Américas". Rev. Bras. Psiquiatria, n. 23, pp. 117-118, 2001.

LOUCURAS que se realizam. "Jornal do Brasil", Rio de Janeiro, 8 de janeiro de 1995. Entrevista com Domingos Sávio.

NÚMERO de internações cai no país, mas especialistas reclamam da falta da verba. Folha de S. Paulo, São Paulo, 18 de maio de 2003. Cotidiano.

ROTELLI, Franco. "A Lei $n$. 180 da reforma psiquiátrica: os problemas na sua aplicação". In: ENCONTRO ÍTALO-BRASILEIRO DE SAÚDE, 18-21 de junho de 1989, Salvador (BA), Saúde mental: a reforma psiquiátrica em questão. Anais ... Salvador, 1989.

SARACENO, B. "Libertando identidades: da reabilitação psicossocial à cidadania possível". Rio de Janeiro: Instituto Franco Basaglia - Te Corá Ed., 1999.

"Il progetto dell'Istituto Mario Negri: Salute Mentale in America Latina". Epidemiologia e Psichiatria Sociale, n. 3, pp. 45-68, 1994

TABORDA, J. G. V.; CARDoso, R. G.; MORANA, Cardoso, H. C. P. "Forensic psychiatry in Brazil an overview". International Journal of Law and Psychiatry, n. 23, pp. 579-588, 2000.

TORREY, E. Fuller. "Jails and prisons: America's new mental hospitals". American Journal of Public Health, n. 5, pp. 1611-1613, 1995.

; MILLER, Judy. "The invisible plague: the rise of mental illness from 1750 to the present". London: Rutgers University Press, 2001, 416 p. WORLD MENTAL HEALTH SURVEY CONSORTIUM. Prevalence, severity, and unmet need for treatment of mental disorders in the World Health Organization World Mental Health Surveys. JAMA, n. 291, pp. 2581-2590, 2004. 